\title{
Anterior Insular-Nucleus Accumbens Pathway Controls Refeeding-Induced Analgesia Under Chronic Inflammatory Pain Condition
}

\section{Grace J Lee}

Seoul National University

Yea Jin Kim

Seoul National University

Kihwan Lee

Seoul National University

Seog Bae Oh ( $\square$ odolbae@snu.ac.kr)

Seoul National University School of Dentistry https://orcid.org/0000-0001-7975-6895

\section{Research}

Keywords: Chronic inflammatory pain, dopamine, insular cortex, nucleus accumbens, refeeding

Posted Date: October 22nd, 2021

DOl: https://doi.org/10.21203/rs.3.rs-955283/v1

License: (c) (i) This work is licensed under a Creative Commons Attribution 4.0 International License. Read Full License 


\section{Abstract}

Feeding behaviors are closely associated with chronic pain in adult rodents. Our recent study revealed that $2 \mathrm{hr}$ refeeding after $24 \mathrm{hr}$ fasting (i.e. refeeding) attenuates pain behavior under chronic inflammatory pain conditions. However, while brain circuits mediating fasting-induced analgesia have been identified, the underlying mechanism of refeeding-induced analgesia is still elusive. Herein, we demonstrate that the neural activities in the nucleus accumbens shell (NAcS) and anterior insular cortex (aIC) were increased in a modified Complete Freund's Adjuvant (CFA)-induced chronic inflammatory pain condition, which was reversed by refeeding. We also found that refeeding reduced the enhanced excitability of alC ${ }^{C a m K I I}$ $\mathrm{NAcS}^{\mathrm{D} 2 \mathrm{R}}$ projecting neurons in this CFA model. Besides, chemogenetic inhibition of alC ${ }^{\mathrm{CamKII}}-\mathrm{NAcS}^{\mathrm{D} 2 \mathrm{R}}$ neural circuit suppressed chronic pain behavior while activation of this circuit reversed refeeding-induced analgesia. Thus, the present study suggests that alC CamKII $-\mathrm{NacS}^{\mathrm{D} 2 \mathrm{R}}$ neural circuit mediates refeedinginduced analgesia, thereby serving as a potential therapeutic target to manage chronic pain.

\section{Introduction}

It has become increasingly clear that feeding behavior affects pain perception. Interestingly, it has been identified that both fasting and food consumption after fasting (i.e. refeeding) suppress pain behavior [1-5]. A recent study reported that hunger-sensitive Agouti-related protein (AgRP) neurons projecting to the parabrachial nucleus (PBN) mediates fasting-induced analgesia in inflammatory pain [6]. We also showed that fasting-induced analgesia is mediated by opioid and endocannabinoid systems [1]. Despite of accumulating evidence supporting for the relationship between fasting and pain reduction, the underlying mechanisms of how refeeding ameliorate pain is still elusive. Since refeeding-induced analgesia includes a variety of factors such as calorie recovery, satiety, and eating behavior [1], the brain circuit mediating refeeding-induced analgesia could be different from that of fasting-induced analgesia. Given that refeeding fulfills physical needs from hunger and provides satisfaction, the brain circuits mediating reward and satisfaction might be implicated in the refeeding-induced analgesia.

The nucleus accumbens (NAc) is a crucial component of the brain reward system [7]. Recent human and animal imaging observations indicate that the dopaminergic system in the NAc is an important neural substrate of feeding behavior and pain perception [8-11]. Indeed, food consumption following $24 \mathrm{hr}$ fasting elevates dopamine release within the NAc for more than two hours [8, 12], but dopamine antagonists cause serious feeding deficit [13]. Also, systemic administration of analgesics enhances dopamine release in the NAc [14] and dopaminergic agonists inhibits nociception [15]. Moreover, several investigations with pharmacological manipulations revealed that dopamine type 2 receptor (D2R) in the NAc plays a critical role in the modulation of pain perception $[16,17]$.

The anterior insular cortex (aIC) is also highly implicated in feeding behavior and pain perception [18-20], and tracing studies in rodents have shown that the NAc receives glutamatergic input from the alC [21]. Functional neuroimaging studies in human also show a correlated activity between the alC and NAc with noxious stimuli [22]. Furthermore, change in functional connectivity between the alC and NAc has been 
also demonstrated along with pain chronification [23]. Given these observations, we hypothesized that the aIC-NAc circuit may underlie refeeding-induced analgesia in the chronic inflammatory pain condition.

In this study, we thus examined whether the alC-NAc circuit is essential for the refeeding-induced analgesia under chronic inflammatory pain condition by employing pharmacological and tract-specific manipulations in a modified complete Freund's adjuvant (CFA)-induced chronic inflammatory pain model.

\section{Materials And Methods}

\section{Experimental animals and pain model}

Both male and female C57BL/ 6 mice and male Fos-tTA-eGFP transgenic mice (Jackson Laboratory, stock \#: 018306) weighing 20-28 g were used for the experiment. The mice were housed 4-6 per cage in a temperature-controlled room $\left(23 \pm 1{ }^{\circ} \mathrm{C}, 12 \mathrm{~h} / 12 \mathrm{~h}\right.$ light/dark cycle) and maintained with pellet diet and tap water ad libitum except for fasting periods. All surgical and experimental procedures were reviewed and approved by the Institutional Animal Care and Use Committee (IACUC, SNU-191001-9-1) at Seoul National University. The experiments were also designed and performed in accordance with the International Association for the Study of Pain (IASP) guidelines. All efforts were made to reduce animal suffering and decrease the number of animals used. For $24 \mathrm{hr}$ fasting, food was removed from the cage but free access to water. For refeeding, mice were given normal chow for $2 \mathrm{hr}$ following $24 \mathrm{hr}$ fasting. We used a modified CFA-induced chronic inflammatory pain model to prolong pain behaviors for more than 20 days, as previously described [24]. $20 \mu \mathrm{L}$ of undiluted CFA (Sigma-Aldrich) was injected subcutaneously into the plantar surface of the left hind with a $0.3 \mathrm{~mL}$ insulin syringe. A booster injection was given 7 days after the first injection. For electrophysiological recordings, the mice were injected with the same amount of CFA into both hind paws. All experiments were performed by a researcher blinded to treatment condition.

\section{Pain behavior tests}

The behavior tests were conducted as described previously [1, 24]. For spontaneous pain measurement, mice were acclimated in the cage at least for a week and then adapted in an acrylic observation chamber (size ranges $12 \times 12 \times 12 \mathrm{~cm}$ ) before the experiment at least three times for three hours. A mirror was located at $45^{\circ}$ angle below the chamber to observe the paws. Mice were video recorded using a video camera for $30 \mathrm{~min}$. The time spent licking or biting was measured by an observer who was blinded to the treatment.

To assess mechanical allodynia, 50\% paw withdrawal threshold was measured using von Frey filaments (North Coast Medical). Mice were acclimated in the cage at least for a week and then adapted in an acrylic cylinder ( $6.5 \mathrm{~cm}$ diameter, $17 \mathrm{~cm}$ height) on the metal mesh floor before the experiment at least three times. All animals were left to an acrylic cylinder on the metal mesh floor for 30 min prior to the mechanical test. The $50 \%$ paw withdrawal threshold was determined based on the up-down method with an ascending series of von Frey filaments $(0.16 \mathrm{~g}, 0.4 \mathrm{~g}, 0.6 \mathrm{~g}, 1 \mathrm{~g}$, and $2 \mathrm{~g})$. 


\section{Administration of drugs}

Sulpiride and SR 141716 were purchased from Tocris. For systemic administration, sulpiride $100 \mathrm{mg} / \mathrm{kg}$ was diluted in 10\% DMSO (Sigma-Aldrich), and $2 \mu \mathrm{L}$ of acetic acid glacial (Duksan) was added, then $\mathrm{pH}$ was adjusted with $\mathrm{NaOH}$ (pH 7.4). For direct brain infusion, sulpiride $(5 \mu \mathrm{g} / \mu \mathrm{L})$ was dissolved in $0.9 \%$ normal saline and unilaterally microinjected $(0.5 \mu \mathrm{L}$ over $5 \mathrm{~min})$ into the contralateral NAcS. Injectors were left in place for an additional 2 min before removal. SR $141716(10 \mathrm{mg} / \mathrm{kg})$ was diluted in $0.9 \%$ normal saline with $10 \%$ DMSO and $1 \%$ tween 80 .

Clozapine-N-oxide (CNO) was purchased from Tocris. For systemic injection, CNO $10 \mathrm{mg} / \mathrm{kg}$ was dissolved in $0.9 \%$ normal saline and intraperitoneally injected 30 min prior to the pain behavior test. For microinjection, CNO $(3 \mu \mathrm{M})$ was dissolved in the $0.9 \%$ saline and administrated into the NAcS 5 min prior to the pain behavior test. For electrophysiology, $\mathrm{CNO}(3 \mu \mathrm{M})$ was dissolved in the bath solution.

\section{Stereotaxic surgeries}

C57BL/ 6 mice were anesthetized with pentobarbital (50 mg/kg, i.p.) and implanted with a guide cannula (26-gauge, $4.6 \mathrm{~mm}$ of length, Plastics One) aimed at the NAcS (coordinates: anteroposterior (AP) 1.4 from bregma, mediolateral (ML) 0.5 from the midline, dorsoventral (DV) -4.6 from skull surface). When inserted, microinjector tips extended $0.5 \mathrm{~mm}$ beyond the guide. The alC (coordinates: anteroposterior (AP) 1.8 from bregma, mediolateral (ML) 2.5 from the midline, dorsoventral (DV) -3.5 from skull surface) was injected with virus.

The following vectors were used for single-site Designer receptors exclusively activated by designer drugs (DREADD) and cre-EGFP expression: pAAV-hSyn-DIO-hM4D(Gi)-mCherry, retrograde pAAV-CaMKIlahM3D(Gq)-mCherry, pENN-AAV-CaMKIla-HI.GFP-Cre-WPRE-SV40 (Addgene). Viral vectors were injected at the rate of $0.2 \mu \mathrm{L} / \mathrm{min}$, with a $10-\mathrm{min}$ diffusion time. The injection volume for the single-site injections was $0.5 \mu \mathrm{L}$. The misplaced cannula or virus injections were excluded from the analysis. DREADD expression was allowed to accumulate for 2 weeks before systemic/local vehicle and CNO injections.

\section{Immunohistochemistry}

Mice were administrated with pentobarbital $(50 \mathrm{mg} / \mathrm{kg})$, then transcardially perfused, first with PBS and then with a $4 \%$ paraformaldehyde solution. Brains were post-fixed at $4^{\circ} \mathrm{C}$ in paraformaldehyde for $1 \mathrm{~d}$ following the extraction and cryoprotected in $30 \%$ sucrose for 3 days. The brains were then sectioned into $40 \mu \mathrm{m}$ slices and preserved in PBS. For the DAB staining, free-floating sections were rinsed in PBS, incubated for $30 \mathrm{~min}$ in $0.3 \%$ hydrogen peroxide PBS solution to quench endogenous peroxidase activity, rinsed several times in PBS, and incubated in a blocking solution that contained $5 \%$ normal goat serum diluted in $0.3 \%$ Triton X-100 in PBS for 60 min. The sections were incubated in rabbit anti-c-Fos polyclonal antibody (1:1000 dilution, ab11137 Abcam) diluted in blocking serum for $48 \mathrm{hr}$ at $4^{\circ} \mathrm{C}$. After incubation in the primary antibody, the sections were rinsed three times for $10 \mathrm{~min}$ in PBS and incubated for $2 \mathrm{hr}$ in biotinylated secondary antiserum made from goat anti-rabbit antibody (1:200 dilution; Vector 
Laboratories) in PBS. Then the sections were processed with ABC kit (PK-6100, Vectastain ABC kit, Vector laboratories) for an hour and visualized with DAB kit (DAB substrate kit for peroxidase, Vector laboratories). After several rinses in PBS, the sections were mounted on coated glass slides, air dried, dehydrated through a series of graded ethanol, and clearing agents, then permounted (Sigma-Aldrich).

To detect D1R, D2R, and CaMKII expression, sections were incubated overnight in blocking buffer $(0.3 \%$ Triton $X-100,2 \%$ bovine serum in PBS) at room temperature. The primary antibodies rabbit anti-D1R (1:200 dilution, ab20066, Abcam), mouse anti-D2R (1:50 dilution, b-10, Santa Cruz), and mouse antiCaMKII (1:100 dilution, sc-5306, Santa Cruz) were diluted in the blocking solution, and the sections were incubated for $48 \mathrm{hr}$ at $4^{\circ} \mathrm{C}$. The slices were washed three times for 10 min each in $0.1 \mathrm{M}$ PBS. To visualize D2R expression, sections were incubated for $2 \mathrm{hr}$ in biotinylated anti-mouse IgG (Vector, PB-9200). Then washed with $0.1 \mathrm{M}$ PBS three times for $10 \mathrm{~min}$ each. The secondary antibody, FITC donkey anti-rabbit (1:200 dilution, 706-095-148, Jackson ImmunoResearch Laboratories), Alexa 488 donkey anti-mouse (1:200 dilution, 715-545-150 Jackson ImmunoResearch Laboratories), Streptavidin (1:200 dilution, s11223, Invitrogen), and DAPI (1:5000 dilution, D9542, Sigma Aldrich) were diluted in the secondary antibody buffer $(0.1 \%$ Triton X-100, 2\% BSA) and incubated $2 \mathrm{hr}$ at room temperature. The sections were then washed three times with $1 \times$ PBS for 10 min each and mounted using Vectashield Mounting media (Vector Laboratories).

\section{Image analysis}

Quantification of c-Fos was performed on slices from bregma +2 to $+1.6 \mathrm{~mm}$ (4-6 sections per mouse, $\mathrm{n}=3$ each group) for alC and bregma +1.4 to $+1.0 \mathrm{~mm}$ ( $4-6$ sections per mouse, $\mathrm{n}=3$ each group) for NAc. Mounted slides were examined under the bright-field microscope (DM5000B, Leica), and images were all taken at $10 \mathrm{X}$ magnification at one time to maintain identical lighting intensity and color balance. Averaged number of c-Fos positive neurons was determined using inverted color images in ImageJ software (National Institutes of Health).

All immunofluorescent-stained sections were imaged on a confocal microscope (LSM 700, Carl Zeiss). We collected 4 sections per mouse ( $n=3$ mice each group), and images were all taken at 200X magnification. Image analysis was performed manually by identifying and counting D1R+, D2R+, CaMKII+ in the same area.

\section{Electrophysiology}

Mice were sacrificed by cervical dislocation and decapitated. The brain was immediately transferred to ice-cold sucrose cutting solution ( $189 \mathrm{mM}$ sucrose, $10 \mathrm{mM} \mathrm{D}$-glucose, $26 \mathrm{mM} \mathrm{NaHCO}_{3}, 3 \mathrm{mM} \mathrm{KCl}, 10 \mathrm{mM}$ $\mathrm{MgSO}_{4} \cdot 7 \mathrm{H}_{2} \mathrm{O}, 1.25 \mathrm{mM} \mathrm{NaH}_{2} \mathrm{PO}_{4}$, and $0.1 \mathrm{mM} \mathrm{CaCl}_{2}$ ), which was bubbled continuously with $95 \% \mathrm{O}_{2}$ and $5 \% \mathrm{CO}_{2}$. The brain was sagittally dissected in a Petri dish containing an ice-cold cutting solution. The brain was glued onto a brain holder, which was placed in a buffer tray containing ice-cold cutting solution. Subsequently, 250- $\mu \mathrm{m}$ sagittal sections were obtained using a vibrating microtome (Leica, VT1200). The sections (slices containing anterior commissure) were then transferred to a chamber on a 
nylon mesh containing external solution (in mM: $124 \mathrm{NaCl}, 3 \mathrm{KCl}, 1 \mathrm{MgSO}_{4} .7 \mathrm{H}_{2} \mathrm{O}, 1.25 \mathrm{NaH}_{2} \mathrm{PO}_{4}, 10$ Dglucose, $24 \mathrm{NaHCO}_{3}$, and $2 \mathrm{CaCl}_{2}$, pH 7.4, osmolality $320-330 \mathrm{mOsm} / \mathrm{kg} \mathrm{H}_{2} \mathrm{O}$ ) bubbled with $95 \% \mathrm{O}_{2}$ and $5 \% \mathrm{CO}_{2}$ at $33^{\circ} \mathrm{C}$. It was incubated for $1 \mathrm{hr}$. The slices could be maintained in a healthy state for up to $8 \mathrm{~h}$ and were transferred to the recording chamber as required.

The slices were continuously perfused with external solution (the same as above) at a rate of 1.0-2.0 $\mathrm{ml} / \mathrm{min}$. The recordings were made at room temperature. The neurons were visualized using infra-red differential interference contrast (IR-DIC) microscopy with a 40x water immersion objective and video imaging camera (BX51Wl, Olympus). Electrophysiological recordings were obtained with a Multiclamp 700B amplifier, Digidata 1330A converter, and pClamp 10.3 software (Molecular Devices), sampled at 20 $\mathrm{kHz}$, and filtered at $2 \mathrm{kHz}$.

Patch pipettes were pulled from borosilicate glass capillaries (OD $1.5 \mathrm{~mm}$, I.D. $0.86 \mathrm{~mm}$; Harvard Apparatus, Edenbridge, United Kingdom) on a horizontal puller (P-97, Sutter Instruments). The pipette tip resistance was 6-8 M . The pipette offset potential was adjusted with the amplifier. The pipettes were filled with an internal solution containing the following components (in mM: 105 potassium gluconate, 20 $\mathrm{KCl}, 10 \mathrm{HEPES}-\mathrm{Na}^{+}$, and $0.1 \mathrm{EGTA}, \mathrm{pH} 7.25$ adjusted with $\mathrm{KOH}$, osmolality $280 \mathrm{mOsm} / \mathrm{kg} \mathrm{H}_{2} \mathrm{O}$ ).

Recordings usually began $>5$ min after obtaining access to the cell. Only one neuron in each slice was exposed to a tested compound a single time, and the slice was replaced after one test on a single cell was performed. The recordings were analyzed with Clampfit 10.7 (Molecular Devices).

\section{Statistical Analysis}

Statistical analysis was performed using GraphPad Prism version 5.0 (GraphPad Software, USA). Comparison between two groups was made using the unpaired Student's t-test. For multiple comparisons, data were analyzed using two-way ANOVA followed by the post hoc Bonferroni test. Detailed statistics for each experiment were shown in the figure legend. Data are presented as mean \pm SEM. Differences with $p<0.05$ were considered significant.

\section{Results}

Systemic D2R antagonist administration reverses refeeding-induced analgesia in chronic inflammatory pain model

Brain dopamine, endocannabinoid and opioid signaling systems play key roles in pain and feeding behavior [25-29]. We first examined the involvement of these neurotransmitters in refeeding-induced analgesia under chronic inflammatory pain conditions via a pharmacological approach with sulpiride (D2R antagonist, $100 \mathrm{mg} / \mathrm{kg}$ ) and SR 141716 (cannabinoid receptor antagonist $10 \mathrm{mg} / \mathrm{kg}$ ). The doses were determined based on the previous studies $[1,30]$, and drugs were intraperitoneally injected after $2 \mathrm{hr}$ refeeding. The chronic inflammatory pain was induced by injecting CFA twice (D 0 initial injection and D 7 booster injection) into the hind paw [24], and behavior tests were conducted at 16 days after the first 
injection (Fig. 1A). The von Frey test and spontaneous pain behavior were measured 30 min after drug injection in the male mice. The result showed that sulpiride reversed refeeding-induced analgesia in both the von Frey test and spontaneous pain behavior test (Figs. 1B, 1C). By contrast, SR141716 did not reverse refeeding-induced analgesia (Fig. S1). We did not examine the involvement of the opioid system in this work because we previously observed that naloxone, a non-specific opioid antagonist, induced a paradoxical analgesic effect in the free-fed group in chronic inflammatory pain conditions [24]. Taken together, our result demonstrated that dopamine signaling is involved in the refeeding-induced analgesia under chronic inflammatory pain conditions.

To determine the sex difference, we repeated behavior tests in the female mice. Similar to the male group, refeeding-induced analgesia was reversed by sulpiride in both von Frey test (Fig. 1D) and spontaneous pain behavior test (Fig. 1E). Since there was no sex difference in the behavior tests, we used the male mice throughout the study.

\section{Refeeding reverses D2R upregulation of the NAcS neurons under chronic inflammatory pain condition}

Based on the involvement of the dopamine system in refeeding-induced analgesia (Fig. 1), we next examined the brain area involved in the refeeding-induced analgesia. Since the dopamine release in the NAc is essential for pain and feeding behavior, we monitored immunoreactivity (IR) of c-Fos, a marker for neuronal activation, in the two subregions of the NAc, which are core (NACC) and shell (NAcS) in male mice [31] (Fig. 2A). Interestingly, the level of c-Fos-IR positive neurons was increased in the NAcS under CFA-induced free-fed group compared to the naïve group, which was significantly decreased by $2 \mathrm{hr}$ refeeding (Fig. 2B). The level of c-Fos expression returned to a similar level of the CFA-induced free-fed group at $24 \mathrm{hr}$ after refeeding (Fig. 2B). In contrast, there was no difference in the level of c-Fos expression within the NAcC between naïve and CFA groups. Also, feeding status did not affect the level of c-Fos expression in the NAcC of the CFA group (Fig. 2B). Taken together, these results demonstrated the involvement of the NAcS in chronic inflammatory pain behavior.

Given that the NAcS contains dopamine receptor type 1 (D1R) and dopamine receptor type 2 (D2R) expressing neurons [32], we next determined c-Fos expressing neurons in the NAcS using male Fos-tTAeGFP transgenic mice. As shown in Figure 2C, we found that the D2R-expressing neurons are highly colabeled with c-Fos rather than D1R-expressing neurons. Also, when we compared the number of D2R IR neurons in the CFA-induced free fed and refed group, D2R IR was significantly decreased in the CFAinduced refed group compared to CFA-induced free-fed group (Figs. 2D, 2E). Together, our results indicate that chronic inflammatory pain led to activation of the NAcS ${ }^{\mathrm{D} 2 \mathrm{R}}$ neurons, which was reversed by $2 \mathrm{hr}$ refeeding.

\section{Sulpiride microinjection into the NAcS reverses refeeding-induced analgesia in chronic inflammatory pain model}

To verify involvement of NAcS ${ }^{\mathrm{D} 2 \mathrm{R}}$ in refeeding-induced analgesia, male mice were microinjected with $\mathrm{D} 2 \mathrm{R}$ antagonist (sulpiride, $5 \mu \mathrm{g} / \mu \mathrm{L}$ ) into the NAcS after $2 \mathrm{hr}$ refeeding (Figs. 3A, 3B). The dose was determined 
based on a previous study [33]. Sulpiride infusion reversed refeeding-induced analgesia in both the von Frey test (Fig. 3C) and spontaneous pain behavior test (Fig. 3D). Taken together, these results imply a critical role of $N A c S^{D 2 R}$ in refeeding-induced analgesia.

\section{The alC CaMKII neurons projecting to NAcS ${ }^{\mathrm{D} 2 \mathrm{R}}$ neurons are activated with chronic inflammatory pain}

The NAcS receives projection from the anterior insular cortex (aIC), which also mediates pain perception and feeding behavior $[18,19,34]$. We hence determined whether the alC is involved in refeeding-induced analgesia. We repeated the c-Fos analysis in the aIC of male CFA group (Fig. 4A) and found that changes in c-Fos expression in the aIC are comparable to that of NAcS. Similarly, the level of c-Fos expression within the alC, was enhanced in the CFA group compared to the naïve group, which was decreased by $2 \mathrm{hr}$ refeeding (Figs. 4A, 4B). The c-Fos expression level returned to a similar level of the CFA-induced free-fed group at $24 \mathrm{hr}$ after refeeding. Overall, these results suggest that the alC also contributes to refeedinginduced analgesia.

It has been shown that the NACS receives glutamatergic input from the alC [21]. Next, we identified target neurons of the alC CaMKII projection within the NAcS by examining D1R and D2R expression. Results showed that $D 2 R$ expressing neurons were co-labeled with mCherry-positive terminals fiber rather than D1R expressing neurons (Figs. 4C-F). These results demonstrate a monosynaptic projection from alC $^{\text {CaMKII }}$ neurons to NAcS ${ }^{\text {D2R }}$ neurons.

\section{Refeeding suppresses excitability of alC ${ }^{\text {CaMKII }}$-innervating NACS ${ }^{\mathrm{D} 2 \mathrm{R}}$ neurons under chronic inflammatory pain condition}

Next, we confirmed the effect of refeeding on the functional activity of the alC CaMKII-NAcS ${ }^{\text {D2R }}$ circuit under chronic pain conditions by whole-cell recordings in the brain slice. As shown in Figures $5 \mathrm{~A}$ and $5 \mathrm{~B}$, we injected pAAV-CaMKIla-hM3D(Gq)-mCherry into the alC of male CFA group and recorded neural spikes in the mCherry expressing NAcS ${ }^{\mathrm{D} 2 \mathrm{R}}$ neurons. In consistent with the previous study [35], spike numbers were increased in the CFA-free fed group (Figs. 5C, 5D). However, spike numbers significantly decreased in the CFA-induced refed group compared to the CFA-induced free-fed group (Figs. 5C, 5D). Also, application of the CNO $(3 \mu \mathrm{M})$ into the bath solution significantly enhanced firings (Figs. 5C, 5D). At the end of each recording, sulpiride $(5 \mu \mathrm{M})$ was applied to confirm that the neurons recorded express D2R. In consistent with the previous study [35], sulpiride application increased the excitability of D2R expressing neurons in the CFA group (Figs. 5C, 5D).

\section{Inhibition of alC CaMKII terminals in NAcS decreases chronic inflammatory pain behavior}

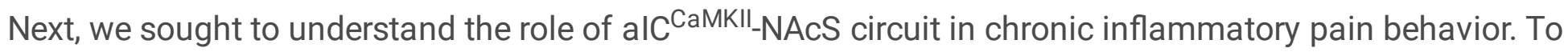
answer this question, we chemogenetically inhibited aIC ${ }^{C a M K I I}-N A C S$ circuit in the male CFA group. The alC was transduced with pENN-AAV-CaMKIla-HI.GFP-Cre-WPRE-SV40 and retrograde pAAV-hSyn-DIOhM4D(Gi)-mCherry was injected into the NAcS (Figs. 6A, 6B). Expression of GFP and mCherry were 
confirmed in the alC via fluorescent imaging (Figs. 6C, 6D), and chemogenetic inactivation by CNO was confirmed by the level of c-Fos expression after systemic CNO injection (Fig. 6E). CNO injection alleviated both mechanical allodynia and spontaneous pain behavior (Figs. 6F, 6G). Together, these results imply that activation of alC ${ }^{\mathrm{CaMKII}}{ }_{-} \mathrm{NACS}^{\mathrm{D} 2 \mathrm{R}}$ circuit is necessary for chronic inflammatory pain behavior.

\section{Activation of alC CaMKII terminals in NAcS reverses refeeding-induced analgesia in chronic inflammatory pain model}

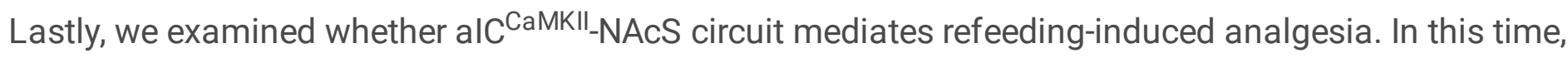
we chemogenetically activated aIC ${ }^{C a M K I I}-N A c S$ circuit following $2 \mathrm{hr}$ refeeding in the male CFA group. Mice were transduced with the hM3Dq receptor via injection of pAAV-CaMKIla-hM3D(Gq)-mCherry, and cannula were placed in the NACS so that CNO could be directly administered to alC terminals (Figs. 7A, 7B). Virus expression was confirmed via fluorescent imaging (Fig. 7C) and chemogenetic activation by CNO was confirmed by the level of c-Fos expression after CNO microinjection (Fig. 7D). CNO infusion reversed refeeding-induced analgesia in both von Frey test (Fig. 7E) and spontaneous pain behavior test (Fig. 7F). These results indicate that activation of alC $^{\mathrm{CaMKII}}-\mathrm{NACS}{ }^{\mathrm{D} 2 \mathrm{R}}$ circuit reverses refeeding-induced analgesia.

\section{Discussion}

We demonstrate in this study that glutamatergic neurons in the alC projecting to the NAcS ${ }^{\mathrm{D} 2 \mathrm{R}}$ mediate refeeding-induced analgesia. Our results indicate that refeeding produces analgesic effect by suppressing neuronal activities of alC ${ }^{C a M K I I}-N A c S^{\text {D2R }}$ circuit, which are enhanced under chronic pain conditions (Fig. 8). Our work thus delineates important neural circuit responsible for refeeding-induced analgesia.

Chronic pain entails structural and functional changes in the mesolimbic system, including the NAc [11, 36-39]. In the previous study, inhibition of the NAcS with lidocaine ameliorated chronic pain behavior but not acute pain behavior[36]. By contrast, inhibition of the NAcC exacerbated acute pain behavior, and optogenetic activation of the NAcC ameliorated chronic pain behavior [40, 41]. These suggest that activation of the NAcS mediates pain perception under chronic pain conditions while activation of the NAcC alleviates pain in both acute and chronic pain conditions. In line with these findings, we showed activated NAcS with pain chronification, which was suppressed by refeeding. These implicate that the NAcS is involved in the refeeding-induced analgesia rather than the NAcC. In the present study, the NAcS was activated under chronic pain conditions and suppressed by $2 \mathrm{hr}$ refeeding, but the NAcC was not affected by refeeding (Fig. 2). These results suggest that the function of the NAcS in regard to pain behavior may alter with pain chronification and may restore by refeeding.

Dopamine release and the level of c-Fos expression increases within the NAcC in response to reward stimuli such as addictive drug administration or refeeding [42-45]. However, reward responsiveness is reduced in chronic pain conditions [46]. Dopamine release induced by sucrose solution intake or morphine as a reward is suppressed [37, 47], and dopamine signaling is reduced in the NAcC under 
chronic pain condition [48]. Similarly, we found that refeeding, a reward stimulus did not increase the level of c-Fos expression within the NAcC under chronic pain condition (Fig. 2B), whereas c-Fos expression increased in response to refeeding in the naïve group (Fig. S2). These findings indicate deficits in the reward-related function of the NAcC under chronic pain conditions, which may result from a hypodopaminergic state. However, dopamine concentration in the NACC needs to be measured in the CFAinduced free-fed and refed group to confirm the dysfunction of the NACC in chronic inflammatory pain. Together, we assume that the reward effect from refeeding may not be involved in the refeeding-induced analgesia.

D1R and D2R expressing neurons in the NAcS play apposite roles in modulating behavior $[49,50]$. Some evidences indicate that D1R is important for reward learning while D2R elicits behavioral aversion [51, 52]. Pain states have been associated with $D 2 R$ in both preclinical animal models and human imaging studies $[11,53]$. A previous study reported that the contribution of $D 2 R$ to anti-hyperalgesia was greater than that of D1R in chronic pain [17]. In another study, chronic pain only elevated D2R protein level, but not D1R protein level [54]. Moreover, a previous study suggests that the intrinsic excitability of the GABAergic NAcS ${ }^{\mathrm{D} 2 \mathrm{R}}$, whose activity suppresses by dopamine binding, was substantially elevated in the chronic neuropathic pain model due to lack of dopamine [35]. Similarly, we showed elevated D2R expression in chronic pain conditions (Figs. 2D, 2E) and enhanced excitability of D2R expressing neurons within the NAcS (Fig. 5D). These findings suggest that the activation of D2R expressing neurons within the NAcS may mediate pain behavior in chronic inflammatory pain, and refeeding may induce analgesia by decreasing its activity. However, further study is needed to clarify the circuits downstream of the NAcS.

Although anatomical connectivity between the aIC and NAcS has been reported [21], the functional connectivity of aIC ${ }^{C a M K I I}-N A c S^{D 2 R}$ is yet to be identified. Consistent with this study [21], we confirmed glutamatergic projection from the alC to the $\mathrm{NAcS}^{\mathrm{D} 2 \mathrm{R}}$ neurons (Figs. $4 \mathrm{E}, 4 \mathrm{~F}$ ) and further revealed functional connectivity through slice patch-clamp recordings. pAAV-CaMKIla-hM3D(Gq)-mCherry was injected into the alC to record neural spikes in the mCherry expressing NAcS ${ }^{\mathrm{D} 2 \mathrm{R}}$ neurons. The excitability of the NAcS ${ }^{\mathrm{D} 2 \mathrm{R}}$ neurons receiving aIC $\mathrm{C}^{\mathrm{CaMKII}}$ projection was increased under chronic pain conditions, but suppressed by refeeding (Figs. 5C, 5D). Moreover, we confirmed the role of aIC ${ }^{C a M K I I}-N A C S^{\text {D2R }}$ circuit in chronic inflammatory pain with chemogenetics. Inhibition of aIC ${ }^{\text {CaMKII-NAcS }}{ }^{\text {D2R }}$ circuit alleviated chronic inflammatory pain behavior (Figs. 6F, 6G) while activation reversed refeeding-induced analgesia (Figs. 7E, $7 F)$. These results imply that activation of aIC ${ }^{C a M K I I}-N A c S^{\text {D2R }}$ circuit may mediate chronic pain behavior. Unlike our results, a previous human fMRI study reported that the functional connectivity of the alC-NAc is decreased in chronic pain [23]. This discrepancy might be due to either difference in subregions of the NAc or differences in the types of pain.

Both chronic pain and feeding behavior involves complex brain circuits that include sensory, reward, and homeostasis $[55,56]$. In this study, we focused on the alC and NAcS, which are known to associate with pain and feeding behavior. However, we do not exclude the possibility that other brain regions may also be participated in the refeeding-induced analgesia, as numerous brain regions interact to process pain 
and feeding behavior $[57,58]$. Indeed, the whole brain c-Fos analysis shows possible involvement of other brain regions such as the basolateral amygdala, medial prefrontal cortex, lateral parabrachial nucleus, and ventromedial hypothalamus [59]. Future studies of different brain regions will further help us to understand the cellular mechanisms and brain circuits for refeeding-induced analgesia.

Refeeding engages multiple features, including eating behavior, calorie recovery, and stomach expansion. It is well elucidated that mastication influences the dopamine release at the pre-synapse in the striatum [60], and glucose-sensing neurons are located in the reward system in the brain [61]. Moreover, the satiety signal that derives from gastric distention and intestinal nutrients that travels to the GLP-1 producing neurons in the NTS, which directly activates the dopaminergic pathways of the mesolimbic system [62]. These findings support our hypothesis that the dopamine system may play an important role in the refeeding-induced analgesia. However, refeeding also engages multiple factors, including systemic hormonal and immune system changes. Imaging studies in the human report that gut hormones such as ghrelin and CCK affect activation of the aIC and NAcS to regulate appetite [63, 64]. Also, it is known that short-term fasting depletes naïve $B$ cells by promoting apoptosis, and the number of naïve $B$ cells is restored by refeeding [65]. Thus, it is highly likely that both the endocrine and immune system are involved in the refeeding-induced analgesia.

\section{Conclusion}

From this study, we demonstrate for the first-time functional significance of alC-NAcS circuit in the modulation of pain behavior. Importantly, this study suggests that modification of feeding behavior can restore altered brain function and induce an analgesic effect in chronic inflammatory pain conditions even without medical treatment. Through verifying a mechanistic understanding for the suppression of chronic inflammatory pain behavior by feeding behavior, our work provides novel therapeutic targets for the development of chronic pain management.

\section{Declarations}

Ethics approval and consent to participate: All experimental protocols received approval from the Institutional Animal Care and Use Committee (IACUC, SNU-191001-9-1) at Seoul National University.

\section{Consent for publication: Not applicable.}

Data and materials availability: All data needed to evaluate the conclusions in the paper are present in the paper and/or the Supplementary Materials.

Competing interests: The authors declare that they have no competing interests.

Funding: This research was supported by a National Research Foundation of Korea grant (NRF2021R1A2C3003334, NRF-2018R1A5A2024418 and NRF-2017M3C7A1025602) funded by the Korean government MSIT (Ministry of Science and ICT). 
Author contributions: G.J.L: designed research, performed most of the experiments, data analysis, figure visualization, wrote the manuscript. Y.J.K: performed cannula implantation. K.L: figure visualization. S.B.O: supervision, funding acquisition, manuscript review \& editing.

Acknowledgments: Not applicable.

\section{References}

1. Lee JY, Lee GJ, Lee PR, Won CH, Kim D, Kang Y, et al. The analgesic effect of refeeding on acute and chronic inflammatory pain. Sci Rep. 2019;9(1):16873.

2. McGivern RF, Berntson GG. Mediation of diurnal fluctuations in pain sensitivity in the rat by food intake patterns: reversal by naloxone. Science. 1980;210(4466):210-1.

3. Hargraves WA, Hentall ID. Analgesic effects of dietary caloric restriction in adult mice. Pain. 2005;114(3):455-61.

4. Liu Y, Ni Y, Zhang W, Sun YE, Ma Z, Gu X. Antinociceptive effects of caloric restriction on postincisional pain in nonobese rats. Sci Rep. 2017;7(1):1805.

5. Jang SP, Park SH, Jung JS, Lee HJ, Hong JW, Lee JY, et al. Characterization of changes of pain behavior and signal transduction system in food-deprived mice. Anim Cells Syst (Seoul). 2018;22(4):227-33.

6. Alhadeff AL, Su Z, Hernandez E, Klima ML, Phillips SZ, Holland RA, et al. A Neural Circuit for the Suppression of Pain by a Competing Need State. Cell. 2018;173(1):140-52 e15.

7. Klawonn AM, Malenka RC. Nucleus Accumbens Modulation in Reward and Aversion. Cold Spring Harb Symp Quant Biol. 2018;83:119-29.

8. Bassareo V, Di Chiara G. Modulation of feeding-induced activation of mesolimbic dopamine transmission by appetitive stimuli and its relation to motivational state. Eur J Neurosci. 1999;11(12):4389-97.

9. Krugel U, Schraft T, Kittner H, Kiess W, Illes P. Basal and feeding-evoked dopamine release in the rat nucleus accumbens is depressed by leptin. Eur J Pharmacol. 2003;482(1-3):185-7.

10. Wood PB. Role of central dopamine in pain and analgesia. Expert Rev Neurother. 2008;8(5):781-97.

11. Taylor AMW, Becker S, Schweinhardt P, Cahill C. Mesolimbic dopamine signaling in acute and chronic pain: implications for motivation, analgesia, and addiction. Pain. 2016;157(6):1194-8.

12. Hernandez L, Hoebel BG. Feeding and hypothalamic stimulation increase dopamine turnover in the accumbens. Physiol Behav. 1988;44(4-5):599-606.

13. Clifton PG, Rusk IN, Cooper SJ. Effects of dopamine D1 and dopamine D2 antagonists on the free feeding and drinking patterns of rats. Behav Neurosci. 1991;105(2):272-81.

14. Navratilova E, Xie JY, Okun A, Qu C, Eyde N, Ci S, et al. Pain relief produces negative reinforcement through activation of mesolimbic reward-valuation circuitry. Proc Natl Acad Sci U S A. 2012;109(50):20709-13. 
15. Morgan MJ, Franklin KB. Dopamine receptor subtypes and formalin test analgesia. Pharmacol Biochem Behav. 1991;40(2):317-22.

16. Moradi M, Yazdanian M, Haghparast A. Role of dopamine D2-like receptors within the ventral tegmental area and nucleus accumbens in antinociception induced by lateral hypothalamus stimulation. Behav Brain Res. 2015;292:508-14.

17. Siahposht-Khachaki A, Pourreza P, Ezzatpanah S, Haghparast A. Nucleus accumbens dopamine receptors mediate hypothalamus-induced antinociception in the rat formalin test. Eur J Pain. 2017;21(7):1285-94.

18. Frank S, Kullmann S, Veit R. Food related processes in the insular cortex. Front Hum Neurosci. 2013;7:499.

19. Bai Y, Ma LT, Chen YB, Ren D, Chen YB, Li YQ, et al. Anterior insular cortex mediates hyperalgesia induced by chronic pancreatitis in rats. Mol Brain. 2019;12(1):76.

20. Wu Y, Chen C, Chen M, Qian K, Lv X, Wang H, et al. The anterior insular cortex unilaterally controls feeding in response to aversive visceral stimuli in mice. Nat Commun. 2020;11(1):640.

21. Gehrlach DA, Weiand C, Gaitanos TN, Cho E, Klein AS, Hennrich AA, et al. A whole-brain connectivity map of mouse insular cortex. Elife. 2020;9.

22. Lutz A, McFarlin DR, Perlman DM, Salomons TV, Davidson RJ. Altered anterior insula activation during anticipation and experience of painful stimuli in expert meditators. Neuroimage. 2013;64:53846.

23. Ikeda E, Li T, Kobinata H, Zhang S, Kurata J. Anterior insular volume decrease is associated with dysfunction of the reward system in patients with chronic pain. Eur J Pain. 2018;22(6):1170-9.

24. Lee GJ, Kim SA, Kim YJ, Oh SB. Naloxone-induced analgesia mediated by central kappa opioid system in chronic inflammatory pain. Brain Res. 2021;1762:147445.

25. Kirkham TC. Endocannabinoids and the non-homeostatic control of appetite. Curr Top Behav Neurosci. 2009;1:231-53.

26. Schultz W. Behavioral theories and the neurophysiology of reward. Annu Rev Psychol. 2006;57:87115.

27. Tuulari JJ, Tuominen L, de Boer FE, Hirvonen J, Helin S, Nuutila P, et al. Feeding Releases Endogenous Opioids in Humans. J Neurosci. 2017;37(34):8284-91.

28. Harris HN, Peng YB. Evidence and explanation for the involvement of the nucleus accumbens in pain processing. Neural Regen Res. 2020;15(4):597-605.

29. Guindon J, Hohmann AG. The endocannabinoid system and pain. CNS Neurol Disord Drug Targets. 2009;8(6):403-21.

30. David V, Durkin TP, Cazala P. Differential effects of the dopamine D2/D3 receptor antagonist sulpiride on self-administration of morphine into the ventral tegmental area or the nucleus accumbens. Psychopharmacology (Berl). 2002;160(3):307-17. 
31. Heimer L, Alheid GF, de Olmos JS, Groenewegen HJ, Haber SN, Harlan RE, et al. The accumbens: beyond the core-shell dichotomy. J Neuropsychiatry Clin Neurosci. 1997;9(3):354-81.

32. Kim J, Park BH, Lee JH, Park SK, Kim JH. Cell type-specific alterations in the nucleus accumbens by repeated exposures to cocaine. Biol Psychiatry. 2011;69(11):1026-34.

33. Anderson SM, Schmidt HD, Pierce RC. Administration of the D2 dopamine receptor antagonist sulpiride into the shell, but not the core, of the nucleus accumbens attenuates cocaine priminginduced reinstatement of drug seeking. Neuropsychopharmacology. 2006;31(7):1452-61.

34. Starr CJ, Sawaki L, Wittenberg GF, Burdette JH, Oshiro Y, Quevedo AS, et al. Roles of the insular cortex in the modulation of pain: insights from brain lesions. J Neurosci. 2009;29(9):2684-94.

35. Ren W, Centeno MV, Berger S, Wu Y, Na X, Liu X, et al. The indirect pathway of the nucleus accumbens shell amplifies neuropathic pain. Nat Neurosci. 2016;19(2):220-2.

36. Chang PC, Pollema-Mays SL, Centeno MV, Procissi D, Contini M, Baria AT, et al. Role of nucleus accumbens in neuropathic pain: linked multi-scale evidence in the rat transitioning to neuropathic pain. Pain. 2014;155(6):1128-39.

37. Kato T, Ide S, Minami M. Pain relief induces dopamine release in the rat nucleus accumbens during the early but not late phase of neuropathic pain. Neurosci Lett. 2016;629:73-8.

38. DosSantos MF, Moura BS, DaSilva AF. Reward Circuitry Plasticity in Pain Perception and Modulation. Front Pharmacol. 2017;8:790.

39. Seminowicz DA, Remeniuk B, Krimmel SR, Smith MT, Barrett FS, Wulff AB, et al. Pain-related nucleus accumbens function: modulation by reward and sleep disruption. Pain. 2019;160(5):1196-207.

40. Magnusson JE, Martin RV. Additional evidence for the involvement of the basal ganglia in formalininduced nociception: the role of the nucleus accumbens. Brain Res. 2002;942(1-2):128-32.

41. Kc E, Moon HC, Kim S, Kim HK, Won SY, Hyun SH, et al. Optical Modulation on the Nucleus Accumbens Core in the Alleviation of Neuropathic Pain in Chronic Dorsal Root Ganglion Compression Rat Model. Neuromodulation. 2020;23(2):167-76.

42. Heffner TG, Hartman JA, Seiden LS. Feeding increases dopamine metabolism in the rat brain. Science. 1980;208(4448):1168-70.

43. Mateo Y, Lack CM, Morgan D, Roberts DC, Jones SR. Reduced dopamine terminal function and insensitivity to cocaine following cocaine binge self-administration and deprivation. Neuropsychopharmacology. 2005;30(8):1455-63.

44. Hamlin AS, McNally GP, Westbrook RF, Osborne PB. Induction of Fos proteins in regions of the nucleus accumbens and ventrolateral striatum correlates with catalepsy and stereotypic behaviours induced by morphine. Neuropharmacology. 2009;56(4):798-807.

45. Wu Q, Lemus MB, Stark R, Bayliss JA, Reichenbach A, Lockie SH, et al. The temporal pattern of cfos activation in hypothalamic, cortical, and brainstem nuclei in response to fasting and refeeding in male mice. Endocrinology. 2014;155(3):840-53. 
46. Schwartz N, Temkin P, Jurado S, Lim BK, Heifets BD, Polepalli JS, et al. Chronic pain. Decreased motivation during chronic pain requires long-term depression in the nucleus accumbens. Science. 2014;345(6196):535-42.

47. Ozaki S, Narita M, Narita M, lino M, Sugita J, Matsumura Y, et al. Suppression of the morphineinduced rewarding effect in the rat with neuropathic pain: implication of the reduction in mu-opioid receptor functions in the ventral tegmental area. J Neurochem. 2002;82(5):1192-8.

48. Selley DE, Lazenka MF, Sim-Selley LJ, Secor McVoy JR, Potter DN, Chartoff EH, et al. Attenuated dopamine receptor signaling in nucleus accumbens core in a rat model of chemically-induced neuropathy. Neuropharmacology. 2020;166:107935.

49. Heiman M, Schaefer A, Gong S, Peterson JD, Day M, Ramsey KE, et al. A translational profiling approach for the molecular characterization of CNS cell types. Cell. 2008;135(4):738-48.

50. Valjent E, Bertran-Gonzalez J, Herve D, Fisone G, Girault JA. Looking BAC at striatal signaling: cellspecific analysis in new transgenic mice. Trends Neurosci. 2009;32(10):538-47.

51. Cole SL, Robinson MJF, Berridge KC. Optogenetic self-stimulation in the nucleus accumbens: D1 reward versus D2 ambivalence. PLoS One. 2018;13(11):e0207694.

52. Hikida T, Kimura K, Wada N, Funabiki K, Nakanishi S. Distinct roles of synaptic transmission in direct and indirect striatal pathways to reward and aversive behavior. Neuron. 2010;66(6):896-907.

53. Mitsi V, Zachariou V. Modulation of pain, nociception, and analgesia by the brain reward center. Neuroscience. 2016;338:81-92.

54. Wawrzczak-Bargiela A, Ziolkowska B, Piotrowska A, Starnowska-Sokol J, Rojewska E, Mika J, et al. Neuropathic Pain Dysregulates Gene Expression of the Forebrain Opioid and Dopamine Systems. Neurotox Res. 2020;37(4):800-14.

55. Talbot K, Madden VJ, Jones SL, Moseley GL. The sensory and affective components of pain: are they differentially modifiable dimensions or inseparable aspects of a unitary experience? A systematic review. Br J Anaesth. 2019;123(2):e263-e72.

56. Liu CM, Kanoski SE. Homeostatic and non-homeostatic controls of feeding behavior: Distinct vs. common neural systems. Physiol Behav. 2018;193(Pt B):223-31.

57. Iannetti GD, Mouraux A. From the neuromatrix to the pain matrix (and back). Exp Brain Res. 2010;205(1):1-12.

58. Song S, Zhang Y, Qiu J, Li X, Ma K, Chen S, et al. Brain structures associated with eating behaviors in normal-weight young females. Neuropsychologia. 2019;133:107171.

59. Lee GJ, Kim YJ, Lee K, Oh SB. Patterns of brain c-Fos expression in response to feeding behavior in acute and chronic inflammatory pain condition. Neuroreport. 2021;32(15):1269-77.

60. Takahashi H. FY, Tsuboi A., Watanabe M., Yamaguchi S. The Influence of Mastication on the Dopaminergic System in the Rat Brain. Interface Oral Health Science 2011 2012:156-8.

61. Koekkoek LL, Mul JD, la Fleur SE. Glucose-Sensing in the Reward System. Front Neurosci. 2017;11:716. 
62. Saper CB, Chou TC, Elmquist JK. The need to feed: homeostatic and hedonic control of eating. Neuron. 2002;36(2):199-211.

63. Zanchi D, Depoorter A, Egloff L, Haller S, Mahlmann L, Lang UE, et al. The impact of gut hormones on the neural circuit of appetite and satiety: A systematic review. Neurosci Biobehav Rev. 2017;80:45775.

64. Blevins JE, Stanley BG, Reidelberger RD. Brain regions where cholecystokinin suppresses feeding in rats. Brain Res. 2000;860(1-2):1-10.

65. Nagai M, Noguchi R, Takahashi D, Morikawa T, Koshida K, Komiyama S, et al. Fasting-Refeeding Impacts Immune Cell Dynamics and Mucosal Immune Responses. Cell. 2019;178(5):1072-87 e14.

\section{Figures}


A

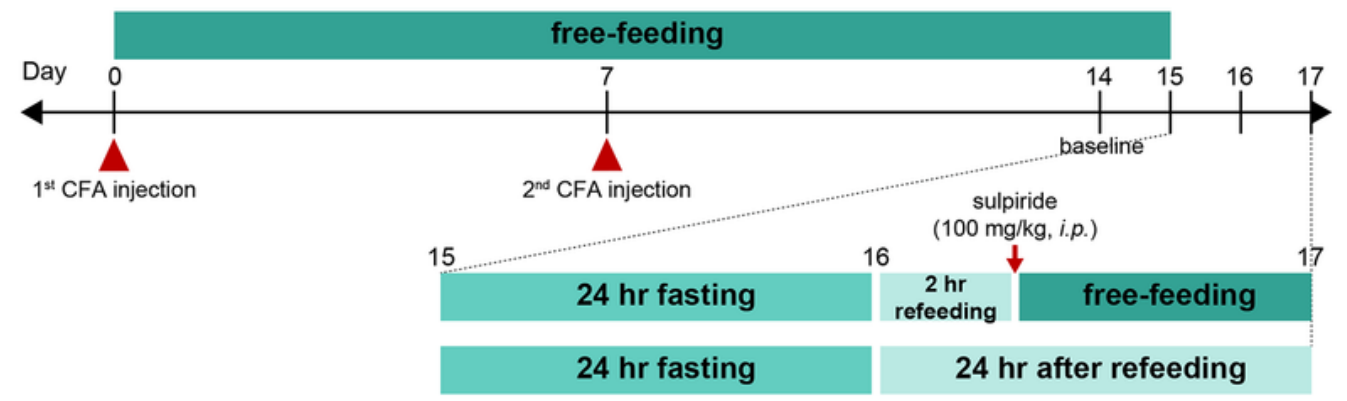

B

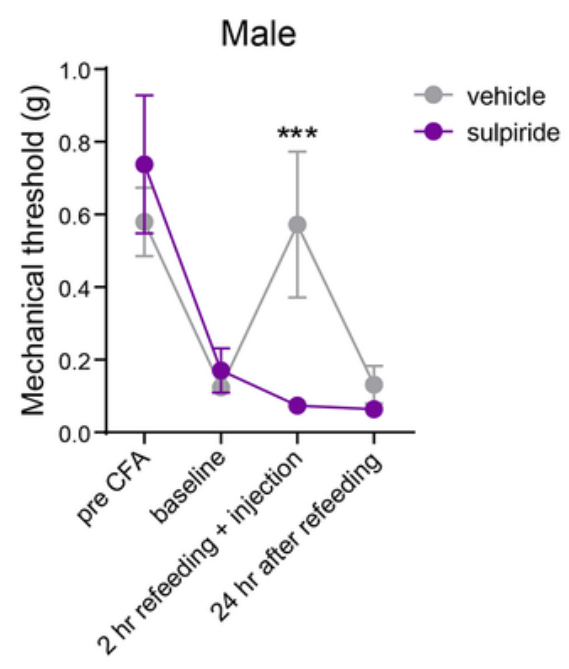

C

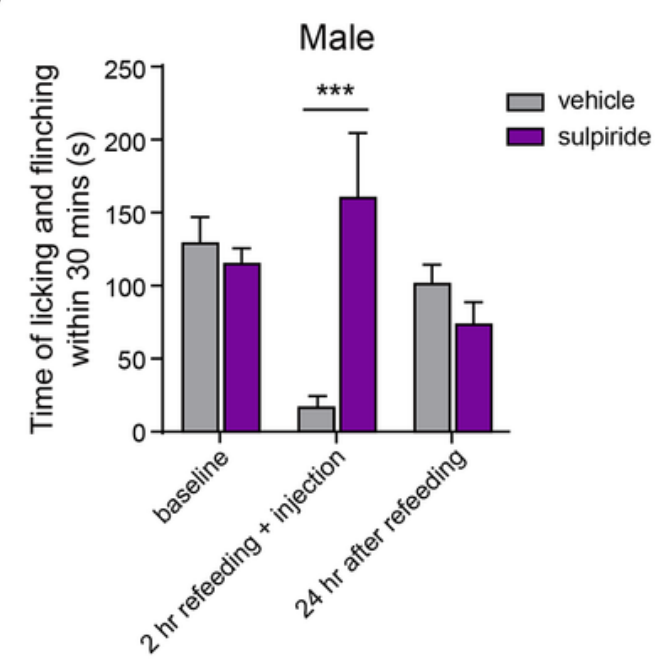

D

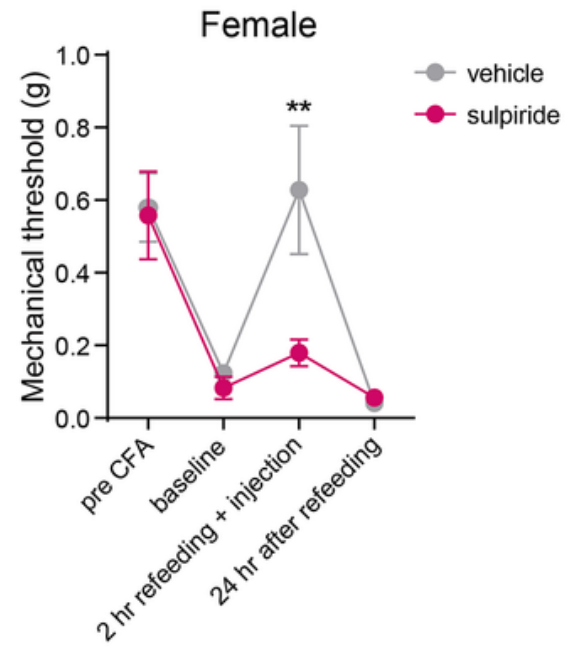

$\mathbf{E}$

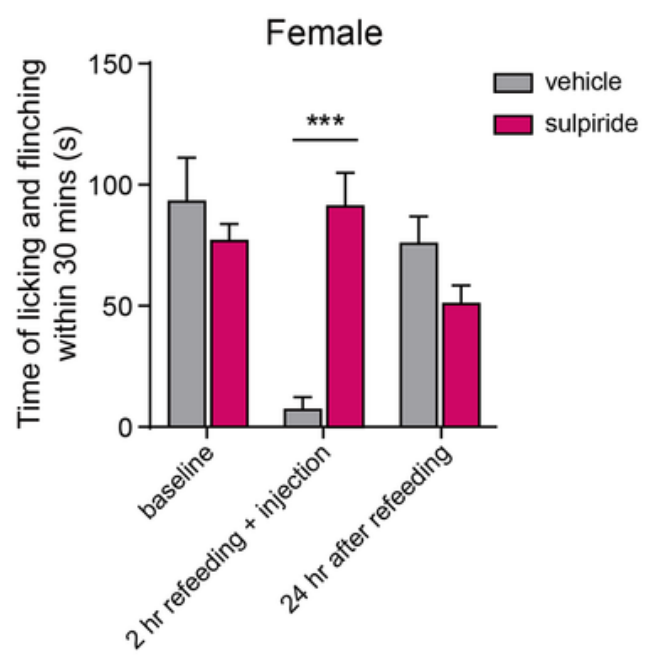

\section{Figure 1}

The effect of D2R antagonist on refeeding-induced analgesia in chronic inflammatory pain model (A) Experimental design and schedule. (B) Effect of D2R antagonist (sulpiride, $100 \mathrm{mg} / \mathrm{kg}$ ) on mechanical allodynia after $2 \mathrm{hr}$ refeeding in the CFA-induced male group. (C) Effect of D2R antagonist (sulpiride, 100 $\mathrm{mg} / \mathrm{kg}$, i.p.) on spontaneous pain behavior after $2 \mathrm{hr}$ refeeding in the CFA-induced male group $(\mathrm{n}=9$ and 7 for sulpiride and vehicle, respectively). (D) Effect of D2R antagonist (sulpiride $100 \mathrm{mg} / \mathrm{kg}$ ) on mechanical 
allodynia after $2 \mathrm{hr}$ refeeding in the CFA-induced female group ( $\mathrm{n}=9$ and 7 for sulpiride and vehicle, respectively). (E) Effect of D2R antagonist (sulpiride, $100 \mathrm{mg} / \mathrm{kg}$, i.p.) on spontaneous pain behavior after $2 \mathrm{hr}$ refeeding in the CFA-induced female group. ${ }^{*} \mathrm{p}<0.01$, ${ }^{* \star} \mathrm{p}<0.001$ (two-way ANOVA followed by Bonferroni's post hoc test)
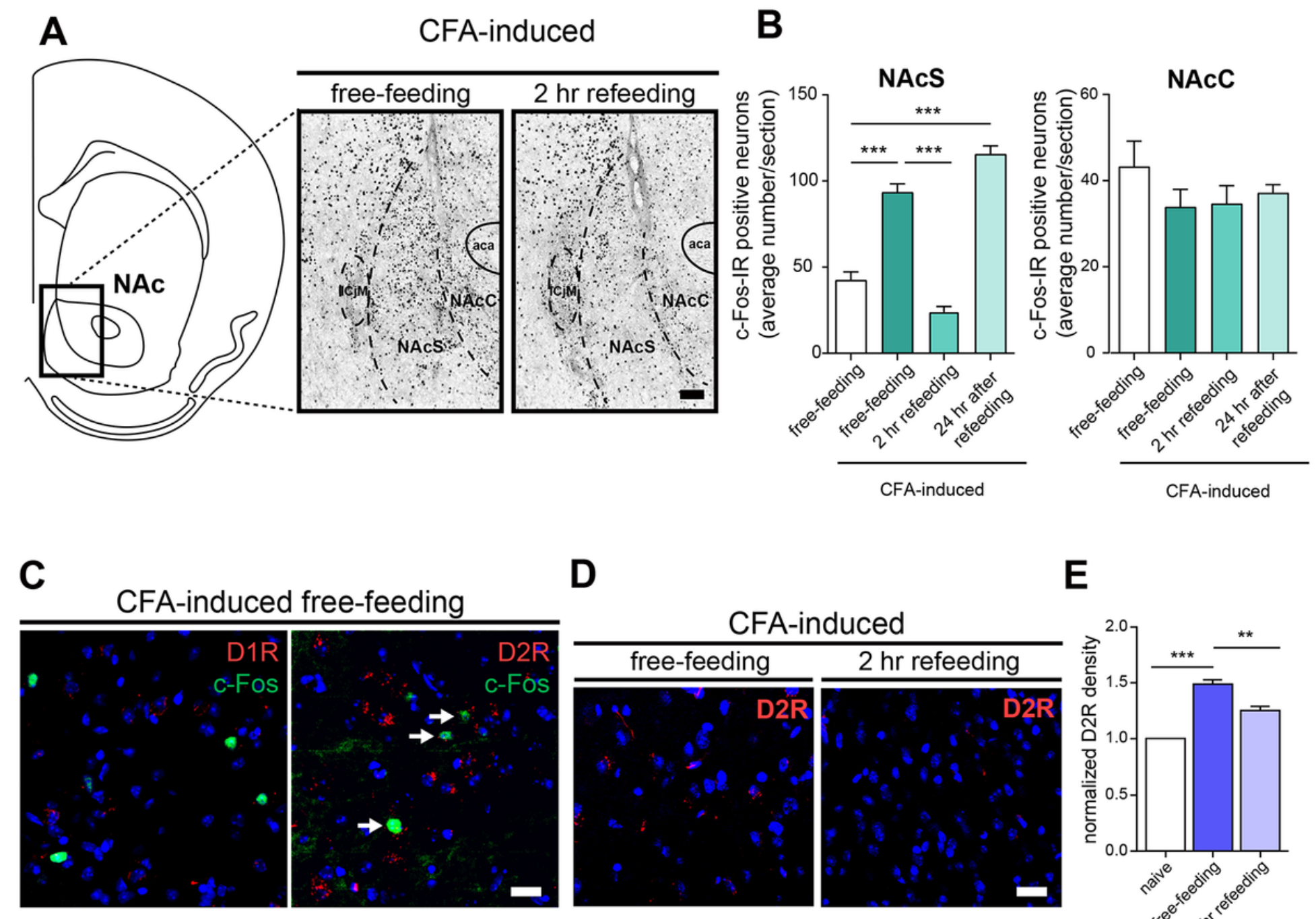

E
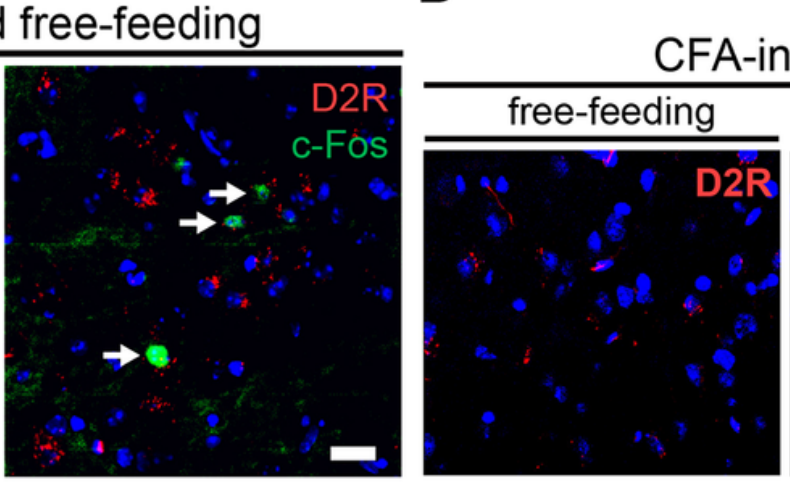

CFA-induced
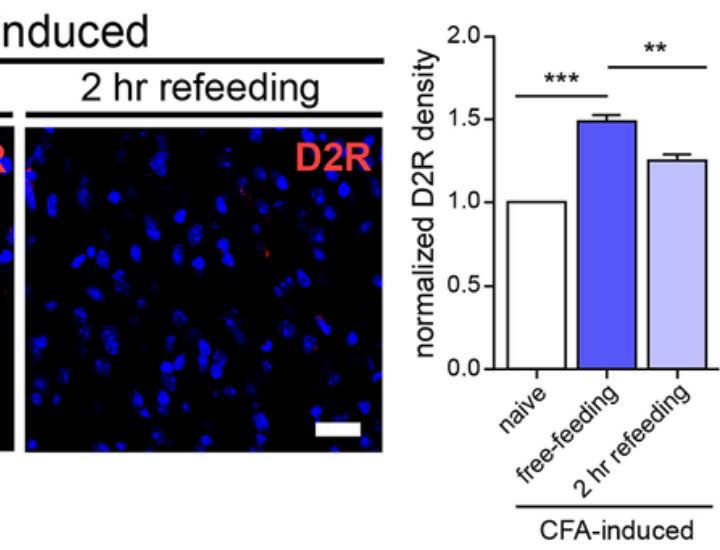

Figure 2

Involvement of NAcS in refeeding-induced analgesia under chronic inflammatory pain (A) Regions of interest in coronal sections based on the mouse brain atlas by Paxinos, George, and Keith B.J. Franklin. Representative c-Fos photomicrographs observed in the NAcC and NAcS. Scale bar represents $100 \mu \mathrm{m}$; magnification 10X. (B) Bar graphs show mean c-Fos positive cell numbers. Data are presented as mean SEM. ${ }^{* \star *} p<0.0001$ (C) Representative images showing c-Fos (green) neurons co-labeled with D1R (left, 
red) or D2R (right red, arrow indicates co-labeled neurons) and DAPI (blue) in the NAcS. Scale bar represents $20 \mu \mathrm{m}$; magnification 200X (1.5). (D) Representative images showing expression of D2R (red) in free fed (left) and refed (right) chronic inflammatory pain group. Scale bar represents $50 \mu \mathrm{m}$; magnification 100X. Normalized density of D2R in the NAcS.
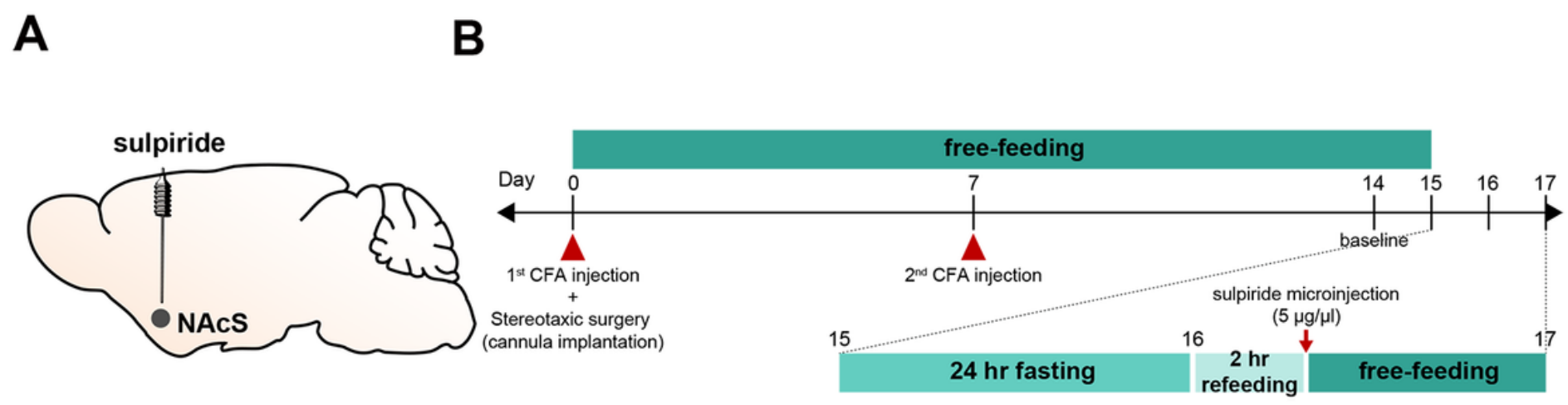

C

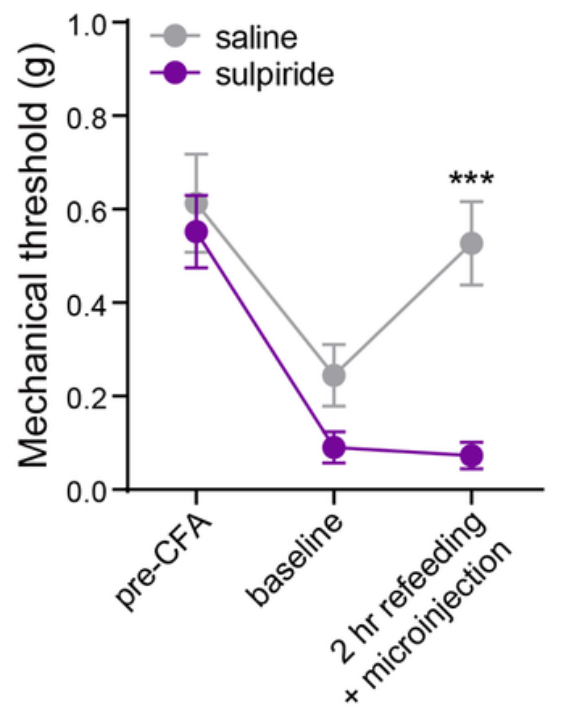

\section{D}

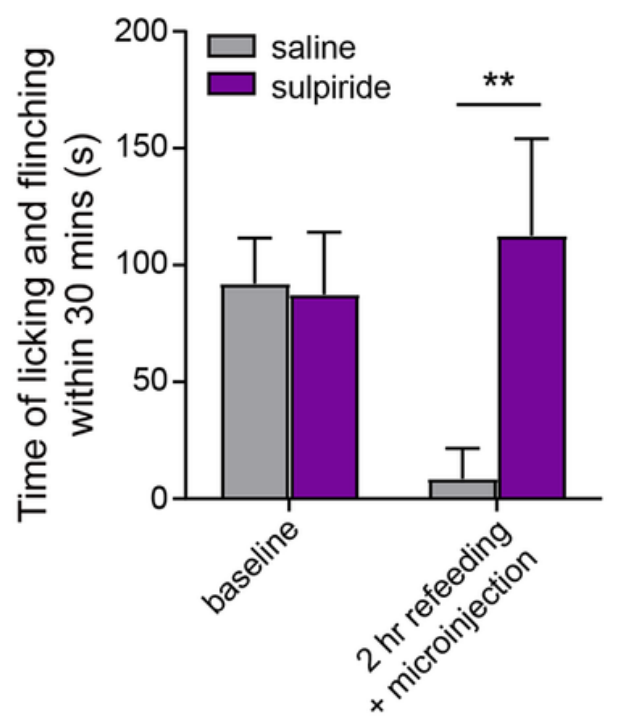

\section{Figure 3}

Effect of sulpiride microinjection into the NAcS on refeeding-induced analgesia (A) Sagittal view of cannula implantation above the NAcS. (B) Schematic diagram of experiment schedule. (C) Mechanical threshold after sulpiride or vehicle infusion in the CFA-induced refed group $(n=8$ and 6 for sulpiride and vehicle, respectively). (D) Time of licking and flinching after sulpiride or vehicle infusion in the CFAinduced refed group ( $\mathrm{n}=8$ and 7 for sulpiride and vehicle, respectively). Two-way ANOVA followed by Bonferroni's post hoc test. Data are represented as mean \pm SEM, ${ }^{* *} p<0.01,{ }^{* \star *} p<0.0001$. 
A

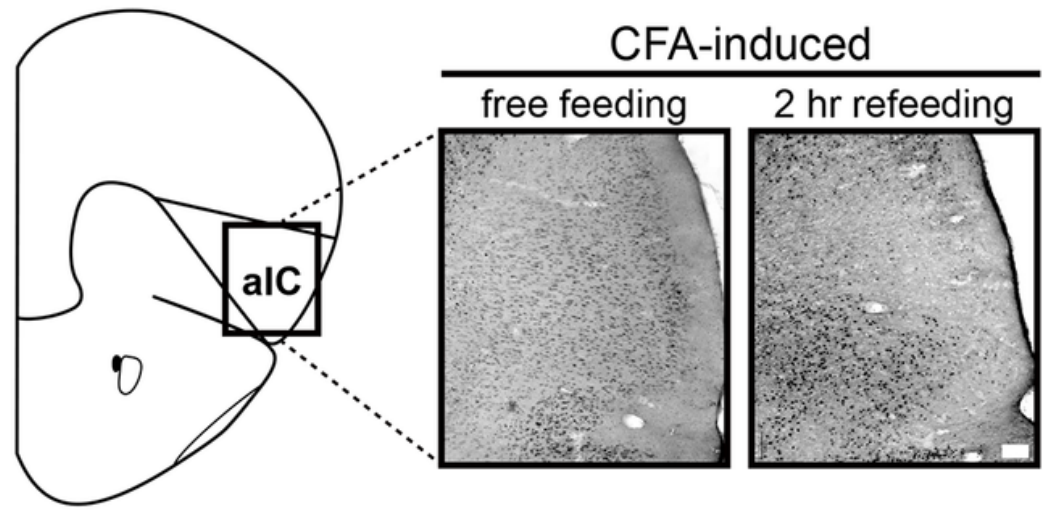

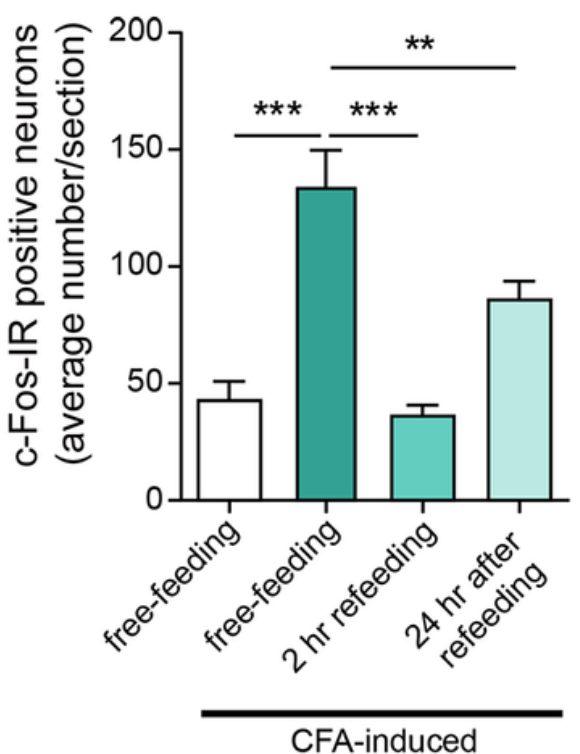

E

$\mathbf{F}$
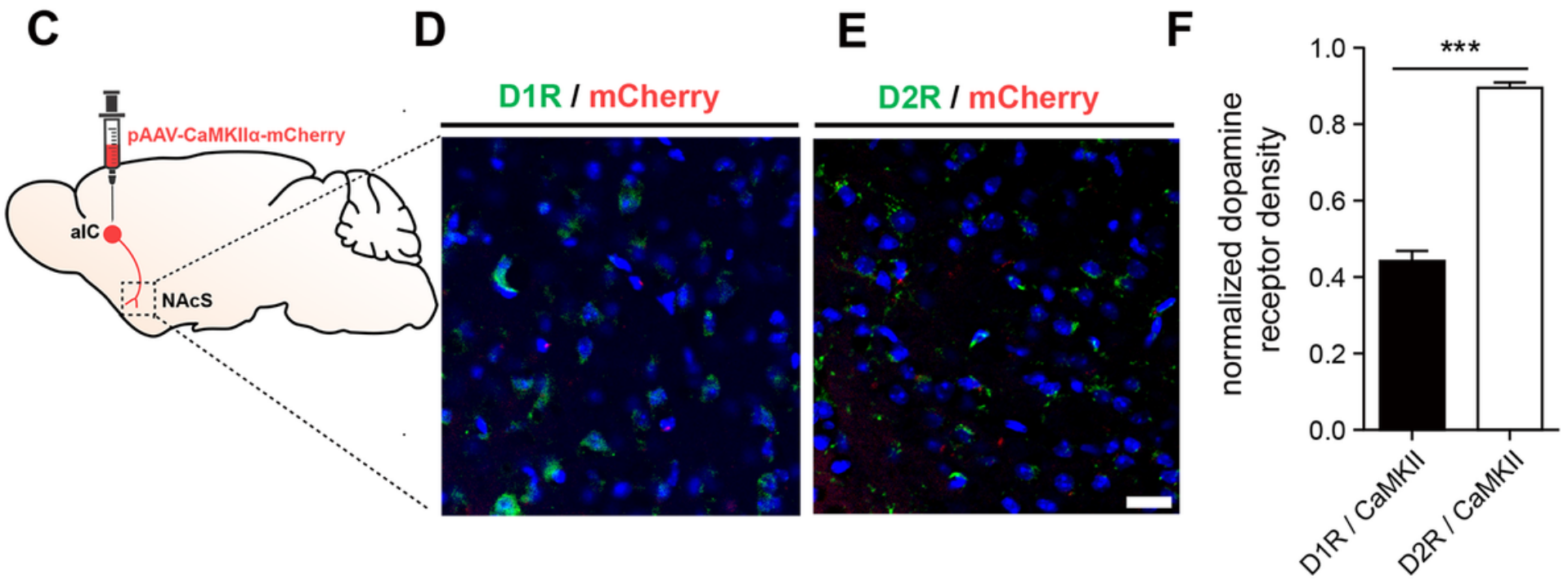

\section{Figure 4}

Involvement of alC in refeeding-induced analgesia under chronic inflammatory pain (A) Regions of interest in coronal sections based on the mouse brain atlas by Paxinos, George, and Keith B.J. Franklin. Representative c-Fos photomicrographs observed in the alC. Scale bar represents $100 \mu \mathrm{m}$; magnification 10X. (B) Bar graphs show mean c-Fos positive cell numbers. (C) Representative images showing c-Fos (green) neurons co-labeled with CaMKII (red) in the alC. Scale bar represents $20 \mu \mathrm{m}$; magnification 200X (1.5). (D) Sagittal view of virus injection into the aIC. (E) Representative images showing hM3D (red) neurons co-labeled with D1R (green) in the NAcS. (F) Representative images showing hM3D (red) neurons co-labeled with D2R (green) in the NAcS. Scale bar represents $20 \mu \mathrm{m}$; magnification 100X (1.5). (G) Normalized D1R or D2R expressing neurons co-labeled with CaMKII. Unpaired t-test. Data are present as mean SEM. ${ }^{* *} p<0.001,{ }^{* \star *} p<0.0001$. 
A

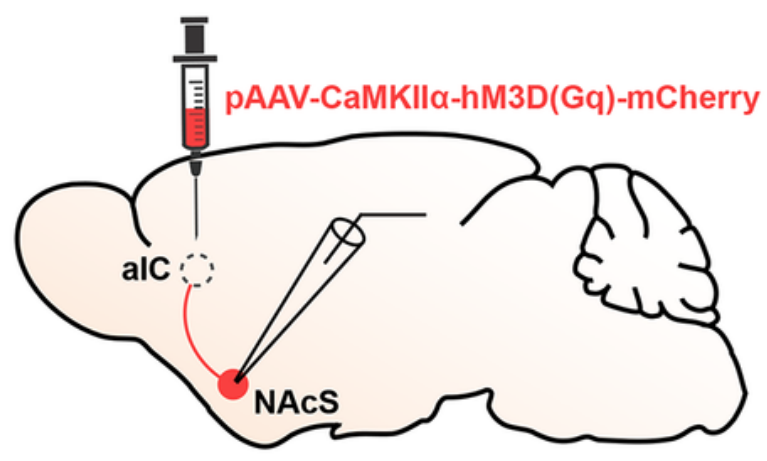

B

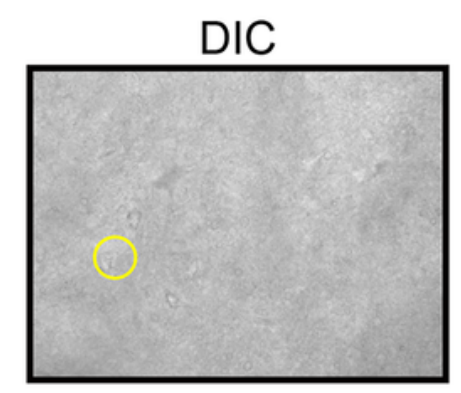

\section{TRITC}

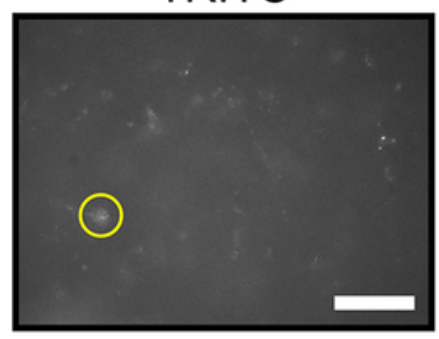

C

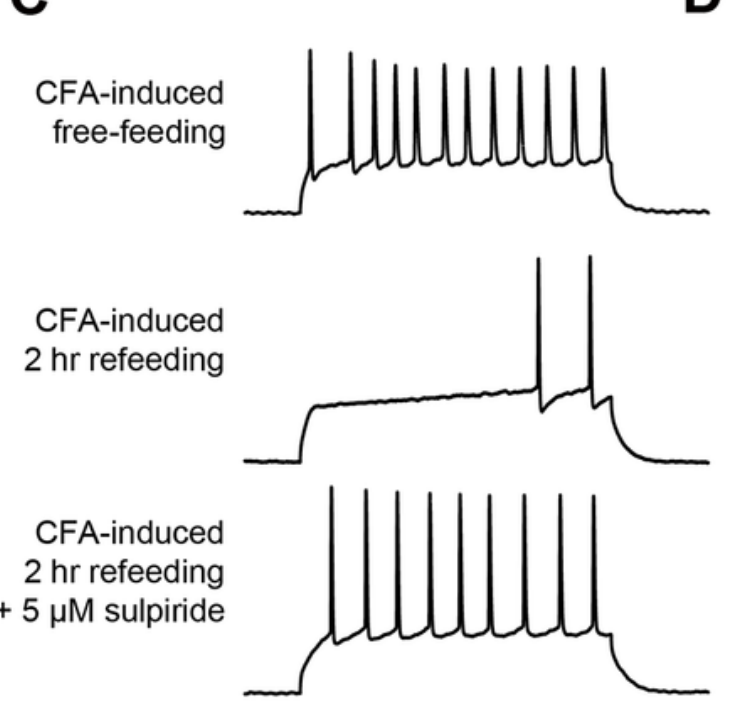

CFA-induced $2 \mathrm{hr}$ refeeding $+3 \mu \mathrm{M} \mathrm{CNO}$

D

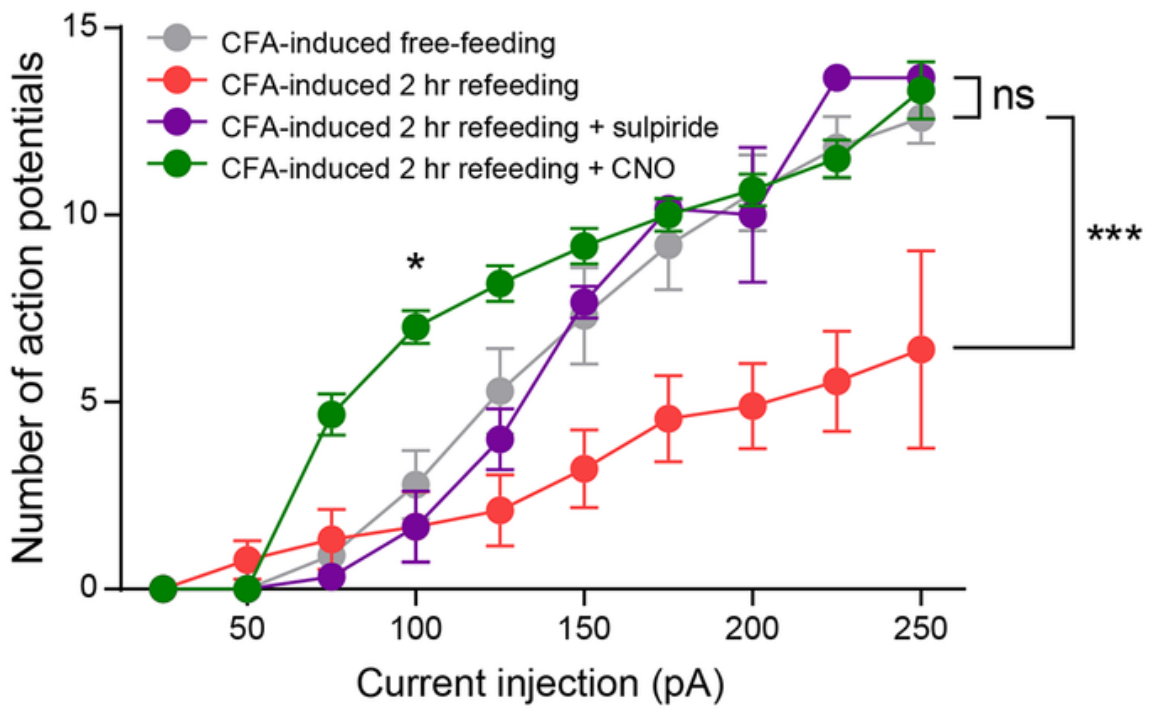

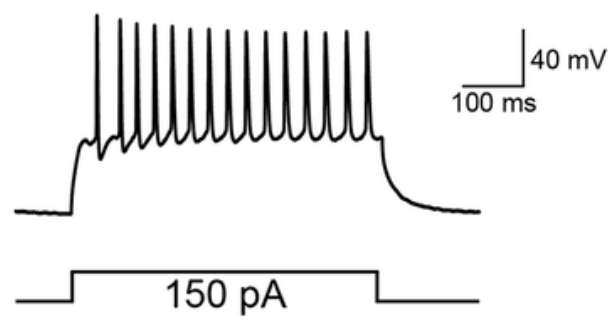

\section{Figure 5}

Effect of refeeding on the excitability of D2R-expressing neurons in the NAcS in inflammatory chronic pain (A) Sagittal view of virus injection into alC and whole-cell patch recording in NAcS. (B) Fluorescent (left) and infrared (right) image of patch-clamp recording from hM3Dq neuron. Yellow circle indicates recorded neuron. Scale bar represents $10 \mu \mathrm{m}$; magnification 40X. (C) Example of current-clamp recording using 150-pA current step in chronic pain model. (D) Mean number of spikes evoked by current steps 
(from 0 to +250 pA in 25 pA increments) ( $n=8$ and 7 for free fed and refed, respectively). Two-way ANOVA followed by Bonferroni's post hoc test. Data are represented as mean $\pm S E M, \star \star p<0.001,{ }^{\star \star \star} p<0.0001$.

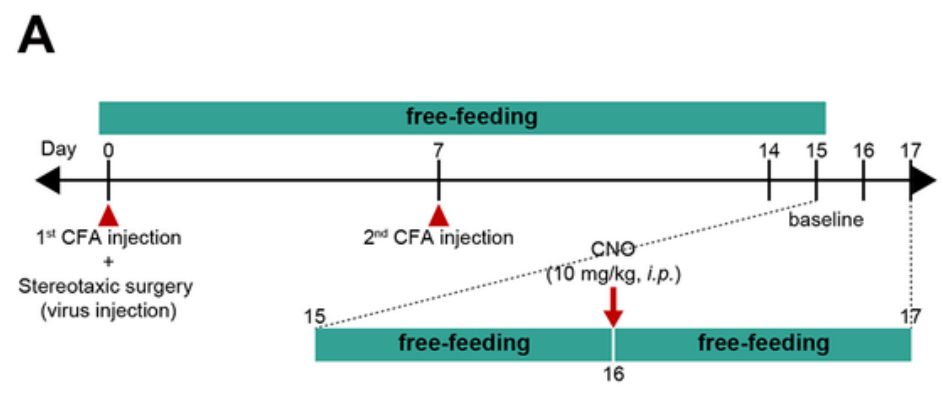

B

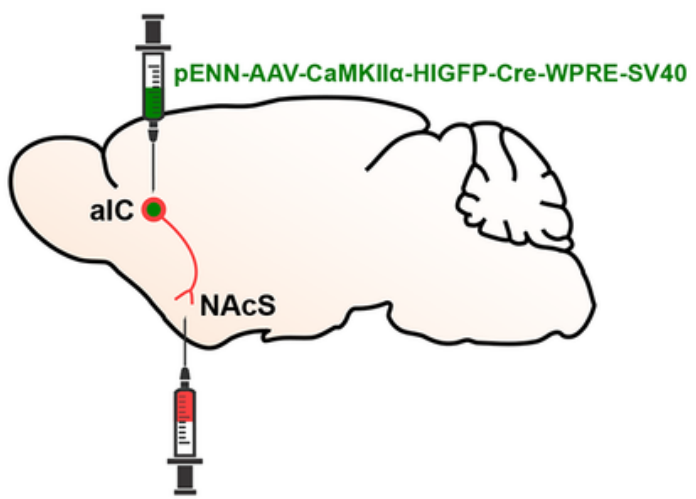

pAAV-hSyn-DIO-hM4D(Gi)-mCherry
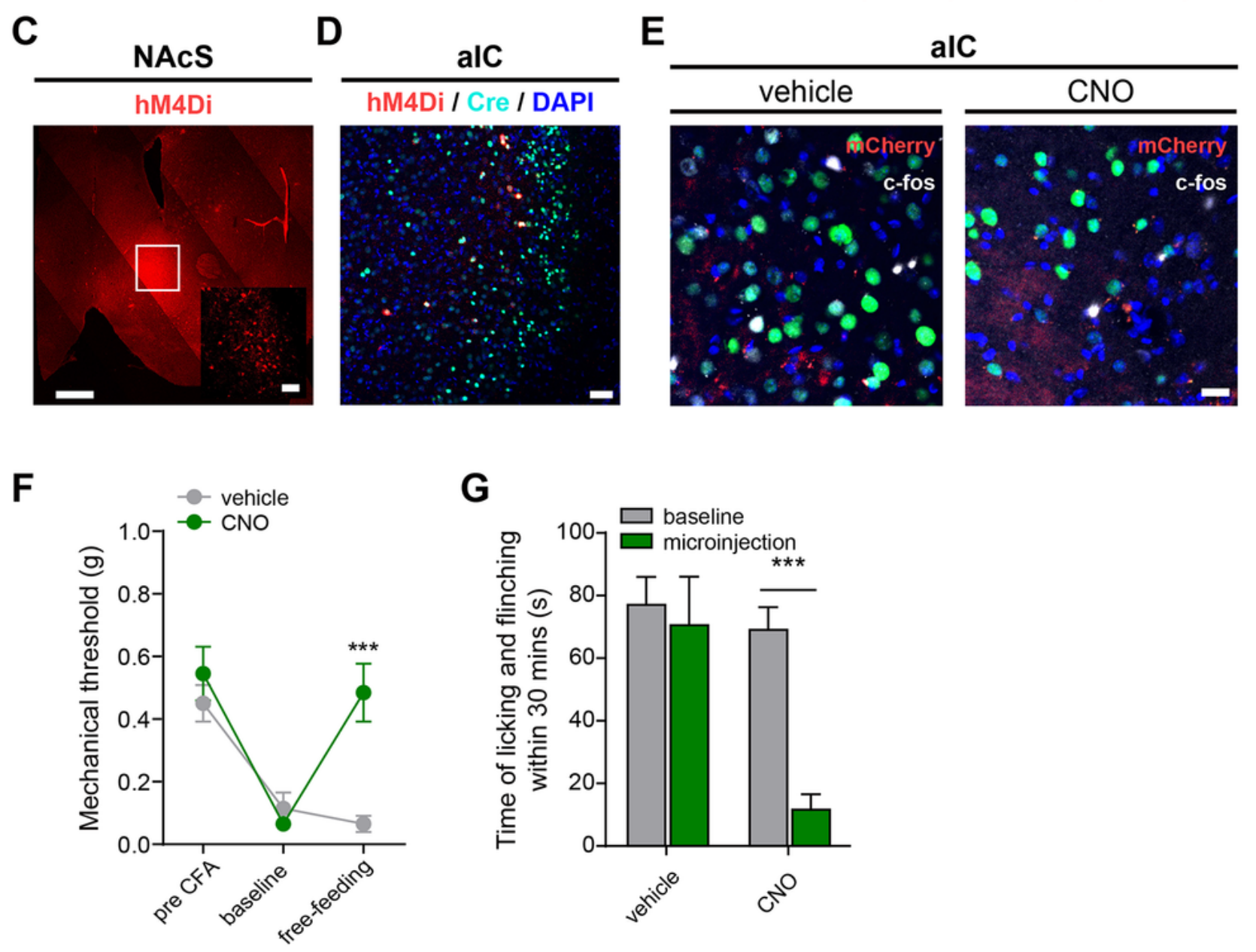

Figure 6

Effect of inhibition of aICCaMKII-NAcS circuit on chronic inflammatory pain behavior (A) Schematic diagram of experiment schedule. (B) Sagittal view of virus injection into the alC and the NAcS. (C) Representative fluorescence image illustrating expression of hM4Di in the NAcS. Scale bar represents 500 
$\mu \mathrm{m}, 20 \mu \mathrm{m}$ in the box; maginification 100X tiled scan and 400X. (D) Representative fluorescence image illustrating expression of hM4Di, Cre, and DAPI in the alC. Scale bar represents $50 \mu \mathrm{m}$. (E) Representative images of c-Fos (grey), Cre (green) and hM3Di (red) expression in the alC that were administered vehicle or CNO. Scale bar represents $10 \mu \mathrm{m}$; magnification 200X (1.5) (F) Change in mechanical threshold in the CFA group after vehicle or $\mathrm{CNO}$ injection ( $\mathrm{n}=9$ and 7 for $\mathrm{CNO}$ and vehicle, respectively). (G) Change in licking and flinching behavior of CFA group after vehicle or CNO injection ( $\mathrm{n}=8$ and 7 for sulpiride and vehicle, respectively). Two-way ANOVA followed by Bonferroni's post hoc test. Data are represented as mean \pm SEM, $* * p<0.01, * \star \star \star p<0.0001$. 
A

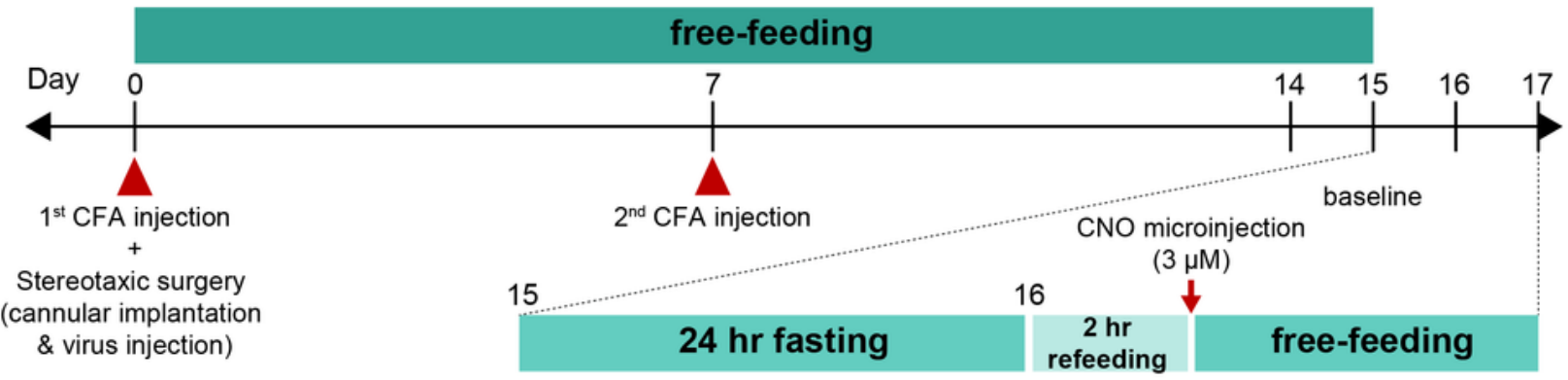

B pAAV-CaMKKII-hM3D(Gq)-mCherry

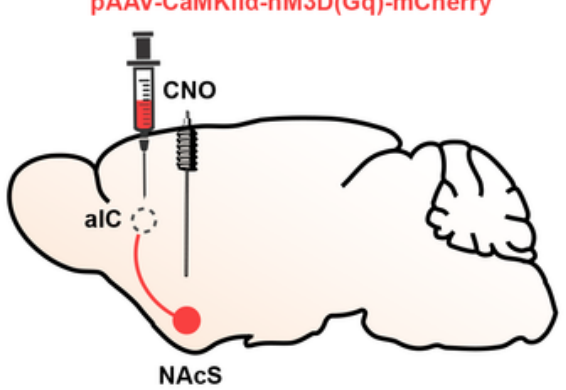

C D NAcS

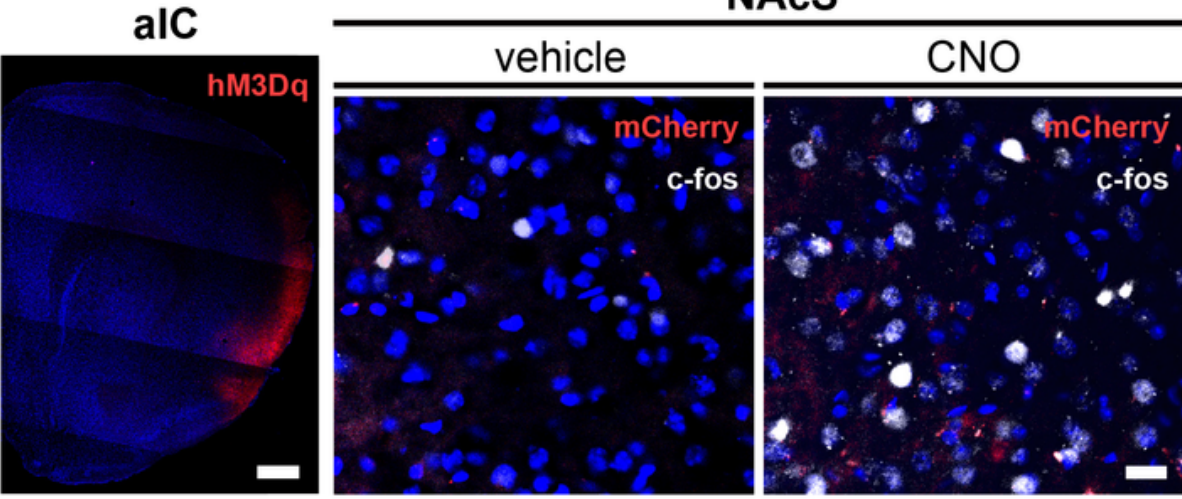

$\mathbf{E}$

$\mathbf{F}$
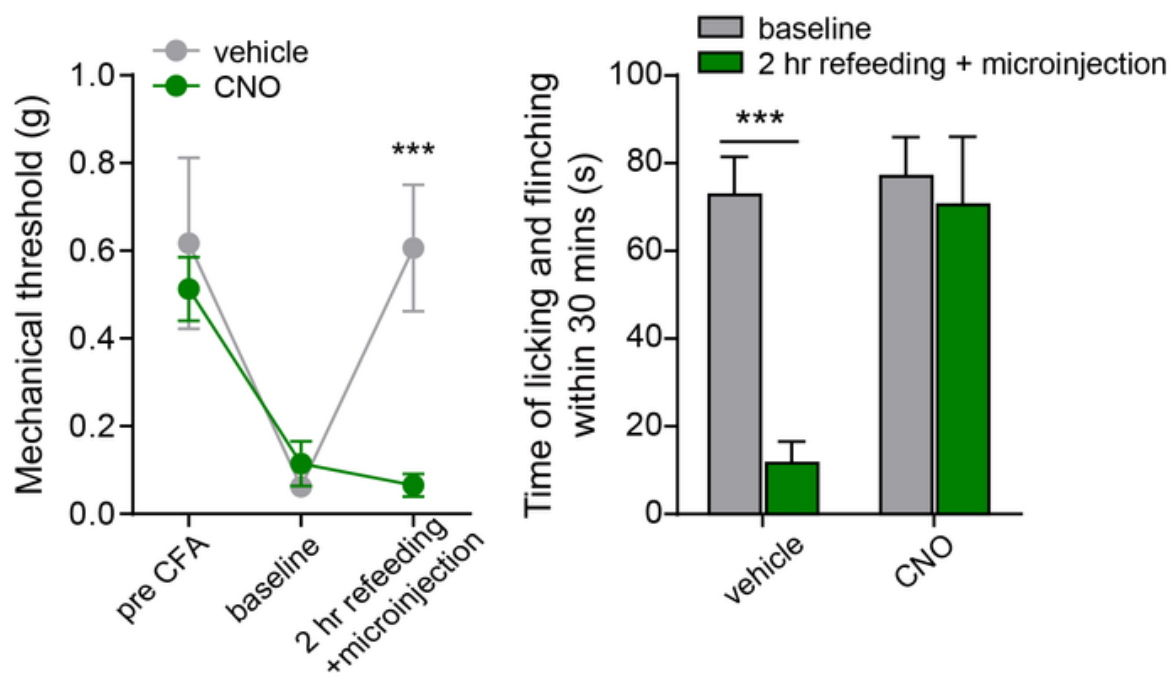

Figure 7

Effect of chemogenetic activation of alCCaMKII-NAcS circuit on refeeding-induced analgesia (A) Schematic diagram of experiment schedule. (B) Sagittal view of virus injection into the alC and cannula implantation above NAcS. (C) Representative fluorescence image illustrating expression of hM3Dq at the injection site. Scale bar represents $500 \mu \mathrm{m}$. (D) Representative images of c-Fos (grey) and hM3Dq (red) expression in the NAcS that were administered vehicle or CNO. Scale bar represents $20 \mu \mathrm{m}$; magnification 
200X (1.5). (E) Change in mechanical threshold with CNO or vehicle infusion in the CFA group $(n=9$ and 6 for sulpiride and vehicle, respectively). (F) Change in time of licking and flinching with $\mathrm{CNO}$ or vehicle infusion in chronic inflammatory pain group ( $\mathrm{n}=10$ and 6 for sulpiride and vehicle, respectively). Two-way ANOVA followed by Bonferroni post hoc test. Data are represented as mean $\pm S E M, * \star p<0.01$, ${ }^{\star \star \star \star} p<$ 0.0001 .

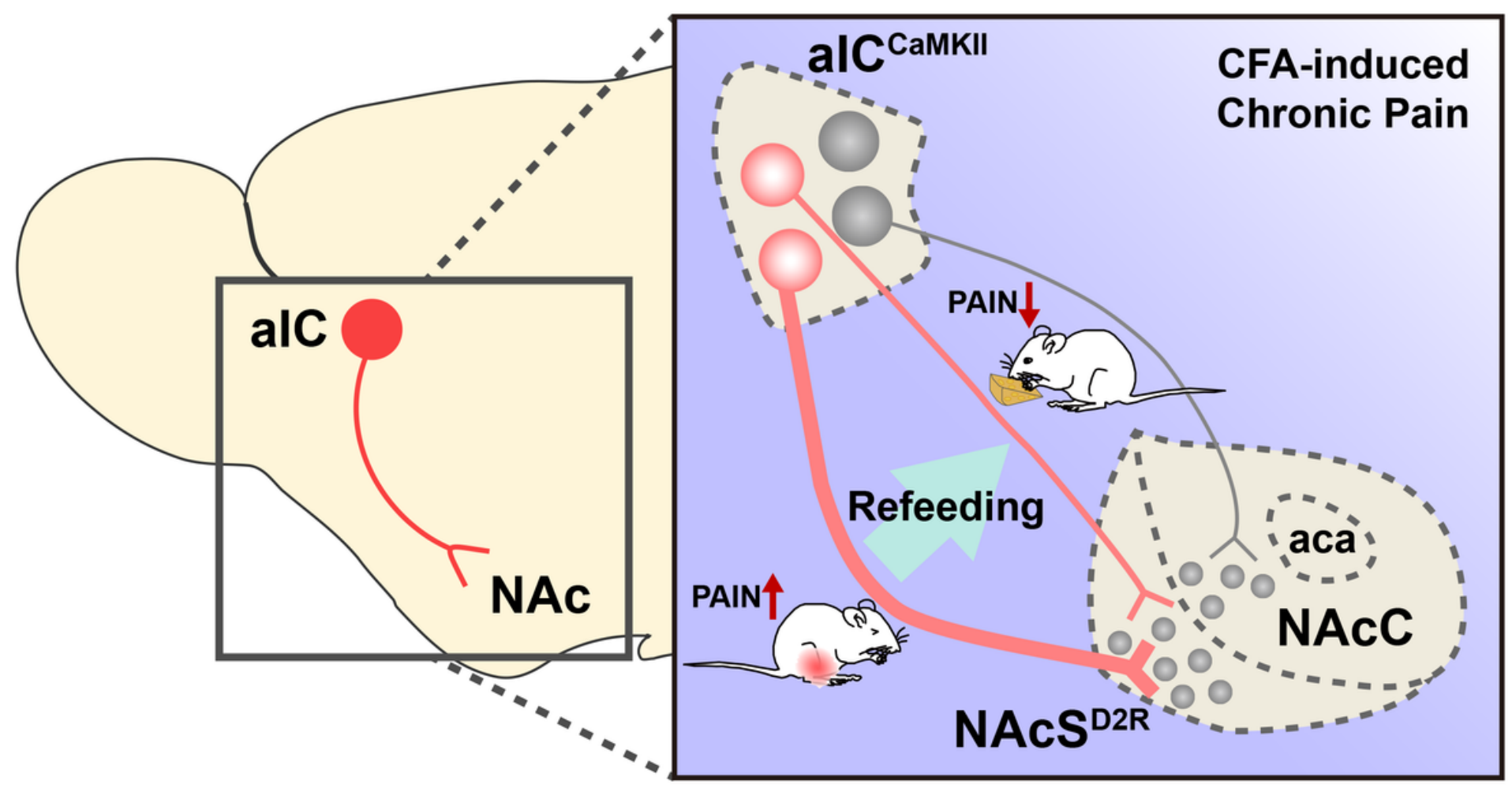

Figure 8

Schematic summary showing that activity of alCCaMKII-NAcS circuit plays a key role in refeedinginduced analgesia

\section{Supplementary Files}

This is a list of supplementary files associated with this preprint. Click to download.

- FigureS1.pdf

- Figures2.pdf 